Article

\title{
« Handicap mental, sexualité et institution : une macro-analyse de la documentation francophone »
}

\author{
Anne-Marie Toniolo, Benoît Schneider et Mélanie Claudel \\ Revue francophone de la déficience intellectuelle, vol. 24, 2013, p. 59-74.
}

Pour citer cet article, utiliser l'information suivante :

URI: http://id.erudit.org/iderudit/1021265ar

DOI: 10.7202/1021265ar

Note : les règles d'écriture des références bibliographiques peuvent varier selon les différents domaines du savoir.

Ce document est protégé par la loi sur le droit d'auteur. L'utilisation des services d'Érudit (y compris la reproduction) est assujettie à sa politique d'utilisation que vous pouvez consulter à l'URI http://www.erudit.org/apropos/utilisation.html

Érudit est un consortium interuniversitaire sans but lucratif composé de l'Université de Montréal, l'Université Laval et l'Université du Québec à Montréal. Il a pour mission la promotion et la valorisation de la recherche. Érudit offre des services d'édition numérique de documents scientifiques depuis 1998.

Pour communiquer avec les responsables d'Érudit : erudit@umontreal.ca 
REVUE FRANCOPHONE DE LA

DÉFICIENCE INTELLECTUELLE

VOLUME 24, 59-74

\title{
HANDICAP MENTAL, SEXUALITÉ ET INSTITUTION : UNE MACRO-ANALYSE DE LA DOCUMENTATION FRANCOPHONE
}

\author{
Anne-Marie Toniolo, Benoît Schneider et Mélanie Claudel
}

\begin{abstract}
La problématique de la sexualité des personnes handicapées mentales se décline en termes de conduites individuelles, pratiques professionnelles et processus organisationnels. Elle est accessible par le biais de sources nombreuses, disparates, hétérogènes qui traduisent l'urgence de préoccupations émanant essentiellement du terrain et de la difficulté de l'évolution conceptuelle et réglementaire à prendre ancrage dans les pratiques. Pour réduire les problèmes inhérents à cette multiplicité d'informations, une analyse est faite de la documentation francophone relative au handicap mental à partir d'un fonds documentaire informatisé constitué à l'occasion de projets de recherche interdisciplinaires. Cette analyse met en exergue les problèmes qui persistent pour rendre la problématique opérationnelle malgré les signes d'évolution.
\end{abstract}

\section{INTRODUCTION}

À la fois reflet et produit d'une lente évolution des mentalités, la définition du handicap et de la dépendance en général vient d'atteindre en France une maturité législative jusqu'ici non égalée. Le cadre conceptuel se trouve renouvelé sur fond d'exigences inédites imposées au terrain (Loi $\mathrm{n}^{\circ}$ 2005-102 du 11 février 2005 pour l'égalité des droits et des chances, la participation et la citoyenneté des personnes handicapées). Plus d'un cinquième de la population totale est susceptible d'être concerné par cette évolution (Rabischong, 2008). Plus de trois millions $(3,4)$ de personnes en situation de handicap mental requièrent une prise en charge (Gardou, 2004) qui ne peut se concevoir que dans le cadre d'une politique nationale de protection sociale. $\mathrm{La}$

Anne-Marie Toniolo, Professeure de psychologie au Laboratoire de Psychologie INTERPSY - Maison des Sciences de l'Homme - Lorraine, Unité de Service et de Recherche - Centre National de la Recherche Scientifique 3261, adresse électronique : toniolo@univnancy2.fr; Benoît Schneider, Professeur de psychologie au Laboratoire de Psychologie INTERPSY - Maison des Sciences de l'Homme - Lorraine, Unité de Service et de Recherche - Centre National de la Recherche Scientifique 3261; Mélanie Claudel, Psychologue au Laboratoire de Psychologie INTERPSY - Maison des Sciences de l'Homme - Lorraine, Unité de Service et de Recherche - Centre National de la Recherche Scientifique 326.

Volume 24, 2013 problématique très actuelle de la sexualité des personnes handicapées mentales est significative de la façon dont ces obligations sociales se déclinent concrètement dans le vécu individuel et collectif.

À partir de cet exemple, elle fait particulièrement bien valoir son impact sur les conduites individuelles, les pratiques professionnelles et les processus organisationnels (Brown, 1997) audelà des frontières de ce territoire national. Elle y parvient parce qu'elle cible une dimension très sensible de l'épanouissement personnel en l'appréhendant de façon exacerbée dans le champ du handicap mental (Delville et Mercier, 1997), là où elle pose le plus d'interrogations en termes de responsabilités, accule la réflexion sociétale dans tous ses retranchements et force ceux qui sont en position de la faire avancer à ne pas s'y dérober (Gascon, 2006; Kerbage et Richa, 2011).

Les approches biologique et sociologique (Giami et De Colomby, 2008) sont devenues insuffisantes pour relever le défi auquel la question est dorénavant confrontée. L'évolution des idées et des obligations sociales ouvre un angle de vue centré sur l'individu, et non plus seulement sur son organisme ou sa catégorie sociale. Cette focalisation prend particulièrement sens lorsque ce dernier est hébergé en institution et que les interactions se complexifient. 
Complémentaire de la biologie et de la sociologie, la psychologie dont l'unité d'étude est précisément l'individu en interaction avec son environnement se trouve propulsée dans le débat, bien au-delà de la compréhension progressiste qu'elle peut apporter au problème. Le cadre national de la législation a aujourd'hui une incidence déterminante sur les pratiques tout en s'articulant fortement aux représentations. Le regard psychologique est en mesure de pointer les difficultés qui surgissent de la confrontation de deux logiques - individuelle et institutionnelle et ce, dans une perspective préventive.

Pour éclairer cette perspective, une approche inductive et descriptive s'impose, car la problématique est accessible par le biais de sources très diverses et disparates. Celles-ci représentent cependant la seule documentation à laquelle peuvent se référer ceux qui veulent ou doivent se pencher sur la question. Elles rendent compte de l'urgence de préoccupations très concrètes émanant du terrain et de la difficulté de l'évolution conceptuelle et réglementaire à prendre ancrage dans les pratiques. Face au foisonnement des informations, un préliminaire est salutaire pour «défricher» ce champ documentaire. Il prend ici la forme d'une macroanalyse qui respecte des critères de classification, sans entrer dans une analyse de contenu à laquelle ne se prêtent pas toutes les formes de support. Cette étape fait valoir la disparité des sources comme symptomatique de la problématique tout en la structurant. Loin d'être toutes de nature « scientifique », ces sources sont rarement abordées dans leur globalité par la communauté scientifique alors qu'elles sont susceptibles d'être consultées par le public et les professionnels en recherche d'informations et de réponses. Aussi, leur variété n'est pas à négliger et leur prise en considération s'avère incontournable.

L'investigation devient alors suffisamment conséquente pour prétendre constituer à part entière matière à communication. Aussi, il s'agira ici de sonder ce prisme et de contribuer par là même à réduire les problèmes inhérents à cette multiplicité d'informations. Pour y parvenir, la démarche s'est appuyée sur un fonds documentaire issu d'un projet de recherche interdisciplinaire visant la sexualité relative à toutes les formes institutionnalisées de dépendance, en se concentrant sur le corpus exclusivement francophone consacré au handicap mental. Ce dernier corpus est un reflet direct des représentations sociales très actives en termes culturels. Il est d'autant mieux en mesure d'en rendre compte que la littérature francophone s'est singulièrement bien exprimée sur la question.

Pour saisir les caractéristiques du corpus sélectionné, nous exposerons les grands axes qui, de l'éthique à la pratique, singularisent la problématique. Ils sont essentiels à rappeler et à comprendre dans la mesure où ils servent de fils conducteurs pour s'orienter dans la variété des sources répertoriées. Nous présenterons les conditions d'élaboration du fonds documentaire qui porte sur la thématique croisée handicap et sexualité dont est extraite la production relative au handicap mental particulièrement ciblée. Nous mettrons en exergue les lignes de force et de faiblesse de cette dernière d'un point de vue quantitatif et qualitatif, en dégageant les indicateurs de son évolution. Cette progression vise à aider les acteurs de terrain à mieux saisir les mouvements de pensées pour structurer l'accompagnement et les chercheurs à mieux positionner leurs paradigmes pour avancer des modèles. Elle permet d'identifier les dynamiques véhiculées par et entre les différents facteurs de progrès (militant, recherche, réflexion éthique et sociétale), de dégager les priorités entre les présupposés du terrain et les questionnements des chercheurs.

\section{HANDICAP MENTAL ET SEXUALITÉ}

La sexualité relève d'une expérience émotionnelle qui concerne la vie affective et relationnelle. Elle est à considérer dans toutes ses dimensions : fonctionnelle (biologique), psychologique (affective, cognitive), sociale, culturelle, juridique, et sous l'angle de ses perceptions, représentations et manifestations (Vaginay et Balvet, 2002). Elle représente une part importante du bien-être et de la qualité de vie. Elle est une affaire de santé, un langage, un rapport au monde, un chemin vers l'intégration et un droit à l'éducation (Giami, 2002). Elle se pose donc comme un droit inaliénable. Dès lors, la question est de savoir ce qu'elle signifie chez une personne dont la conscience est entravée et qui doit passer sa vie sous le contrôle permanent d'une institution. 


\section{$\underline{\text { Une question d'éthique }}$}

D'emblée la question de la sexualité des personnes en situation de handicap s'inscrit comme la pierre angulaire d'un raisonnement éthique (Agthe Diserens, Mercier et Vatre, 2002). Bien au-delà de toute considération scientifique, elle renvoie à la question de savoir jusqu'où une société et chacun de ses membres sont prêts à reconnaître comme être à part entière toute personne rendue vulnérable du fait d'une perte ou d'un manque d'autonomie liés à son état physique, psychique ou mental. La réponse est loin de s'imposer immédiatement à la raison et demeure souvent ambiguë. Les convictions intellectuelles, l'empathie et/ou la bonne foi guidées par l'émotion sont vite ébranlées par la ténacité de représentations bien enfouies qui refont vite surface dès qu'elles sont activées par des interpellations délicates. La sexualité est l'une de ces interpellations singulièrement révélatrice d'une telle adhésion si fragile, alors même qu'elle participe à la construction identitaire.

En matière de sexualité, ce qui est vrai pour tout handicap l'est particulièrement lorsqu'il est mental (Delfosse, Delville, Duchene, Mattys, Mercier et Witd, 1997). Considérées comme asexuées ou gouvernées par une sexualité déviante (Giami, Humbert et Laval, 2001), les personnes concernées ont vu leur dimension sexuée réprimée ou largement occultée dans l'histoire de leur prise en charge, y compris dans ses épisodes très récents. Alors qu'elles partagent avec tout un chacun le désir d'être aimées et d'aimer (Diederich et Printz, 2000), elles ont fréquemment subi de fortes pressions leur interdisant toutes relations sexuelles et même affectives. Considérées comme «anormales» et/ou représentant un poids social, incapables d'assumer toute responsabilité parentale, la société a pu faire le choix de leur éradication à une époque d'égarements dramatiques. À d'autres moments, elle a recouru à des voies autrement radicales (Gasser, Heller et Jeanmonod, 2004) encore d'actualité en France en 1996, lorsque le Comité national d'éthique publie deux rapports restés sans suite où il examine la possibilité de légalisation de la stérilisation comme méthode contraceptive.

\section{Intimité et consentement}

La sexualité se double d'un corollaire l'intimité — définie comme ce qui n'est pas révélé aux autres. Révéler cette part de soi-même fragilise, dans la mesure où le destinataire peut exercer son emprise sur cette dimension exposée. Seule une relation de confiance avec le bénéficiaire permet d'écarter tout pouvoir d'emprise. Le besoin d'intimité pour soi-même et la perception de son existence chez les autres s'acquièrent progressivement. Vaginay (2006) rappelle que cette exigence procède d'un apprentissage, celui qui distingue vie publique et vie privée. Partant, un consensus collectif implicite se construit selon lequel chacun finit par respecter cette part de l'autre qui peut attirer ou intriguer, donner envie à soi ou à l'autre de la dévoiler. Entre séduction et sentiment amoureux la démarche est alors possible sous réserve d'un rapport équilibré et consenti, alors que sans consentement, toute irruption dans l'intimité de l'autre peut basculer dans l'atteinte à la pudeur et le viol. Cette notion de consentement devient ainsi la charnière des processus en jeu dans cette vulnérabilité singulière.

Le handicap mental met en cause l'accès au libre consentement (Bernardet, 2004). La fragilité est ici celle de personnes qui n'ont pas entièrement ou pas du tout connaissance de la valeur de leurs actes et qui deviennent potentiellement des objets dont on peut abuser. Pour les préserver des abus auxquels leur vulnérabilité les expose, la législation assure en France une protection fondée sur le code civil (Livre ${ }^{\mathrm{er}}$ : Des personnes; Titre XI : De la majorité et des majeurs protégés par la loi). Cette protection est confirmée par la loi du 5 mars 2007 portant sur la réforme de la protection juridique des majeurs qui renforce le principe de respect de l'autonomie personnelle. Ainsi, celui qui protège la personne handicapée mentale n'a pas le droit de faire obstacle à sa sexualité. La notion de consentement permet de qualifier juridiquement un acte. Mais consentir à un acte suppose avoir la lucidité pour exprimer un consentement libre et éclairé.

\section{Le poids des représentations}

Pendant longtemps, pour des raisons sociologiques et de puritanisme, la morale ne reconnaissait la sexualité que liée à la procréation sous le seul sceau du mariage (Germain et Langis, 1990). Le handicap mental n'autorisant 
pas l'autonomie pour envisager le mariage, la question de la sexualité pouvait trouver légitimité à être évacuée en toute bonne conscience de la réflexion collective. Depuis, une nouvelle posture sociétale a pris place qui dissocie sexe, amour et procréation. Cependant, cet espace de liberté n'est pas sans paradoxes. Par exemple, la sexualité libérée des sentiments est autorisée à s'exprimer par l'érotisme. Mais ce dernier n'est magnifié que dans l'union de corps parfaits. Dans le même ordre d'idée, alors même que l'état favorise la reconnaissance de la personne en situation de handicap, les progrès médicaux ouvrent la voie à la sélection de l'embryon avec en arrière-fond le mythe d'un individu parfait, ici, dans sa totalité. Ces ambiguïtés entretiennent de nombreux freins qui vont à l'encontre de l'idée que des esprits altérés et des corps malformés peuvent accéder au désir, au plaisir et à l'amour (Mercier, 2004)

Victimes de représentations individuelles et sociales dévalorisantes (Lavigne, 1996), les personnes handicapées mentales sont l'objet de processus d'infantilisation qui renforcent considérablement leur dépendance. Leur accompagnement est freiné par la difficulté de les concevoir comme adultes (Dupras, 1998), de les projeter dans l'avenir. Elles souffrent de surinvestissement affectif de la part des parents qui peinent à admettre que, malgré ses limites intellectuelles, leur enfant a grandi. Elles sont invitées à appréhender leur propre sexualité sous l'angle de sa fonction strictement biologique, dans la mesure où pour leurs proches elle est la seule version acceptable, car irréfutable. Cette perception explique la difficulté pour les intéressés d'érotiser leurs désirs et de concevoir un projet de vie avec un(e) autre, et se trouve ébranlée lors du changement physiologique qu'opère en eux la maturité sexuelle. La situation devient alors particulièrement sensible pour la femme. Perçue jusque là comme un risque abstrait, la maternité devient possible et le franchissement de cette étape signifie pour ceux qui l'entourent l'angoisse de la procréation.

\section{LA PLACE DU MILIEU INSTITUTIONNEL}

Ces trois piliers de la problématique (éthique, consentement et représentations) s'articulent obligatoirement en milieu institutionnel de par l'obligation faite aux établissements de s'adapter aux évolutions du cadre juridique qui régissent leurs missions et leur fonctionnement. Les lois ont évolué en intégrant de plus en plus ces trois notions. Il revient au système institutionnel d'être conforme à ces textes. Le système institutionnel joue un rôle d'autant plus pivot du fait de l'importance de l'effectif et de la dépendance des personnes accueillies, et de son positionnement dans l'accompagnement. Or, la question de la sexualité est véritablement abordée depuis peu dans les établissements au sein desquels elle provoque des remous au regard des situations nombreuses et variées qui engagent la responsabilité des proches et des professionnels.

\section{Des facteurs favorisants}

En France, en 2006, les adultes déficients intellectuels et psychiques représentent plus de $70 \%$ des personnes hébergées, essentiellement en internat $(38 \%)$ ou en accueil de jour $(46 \%)$ (Enquête Handicap, Incapacités, Dépendances, 2006). Ces chiffres signifient que pour ces adultes l'intégration en milieu ouvert a rencontré des limites. De 1995 à 2006, le nombre de structures et les places pour les personnes lourdement handicapées a fortement augmenté en réponse à une demande jusque là insatisfaite, $\mathrm{du}$ fait du vieillissement des personnes accueillies et de l'attente des parents qui ne peuvent plus s'occuper d'elles. Ainsi, l'institution prend de plus en plus le relais des familles et se trouve par là même de plus en plus interpellée sur la question de la qualité de vie qu'elle est censée offrir.

Cette prise en compte est en fait liée à la reconnaissance lente et progressive de l'individu et de sa participation dans la gestion de son handicap au sein des établissements. Pendant la première moitié du $20^{\mathrm{e}}$ siècle, ces derniers dépendaient avant tout d'œuvres charitables cautionnant la bonne conscience des pouvoirs publics et de la société. Le souci de la gestion collective primait alors les besoins individuels. Assistés, les résidents n'avaient aucun droit à la parole. Après la Seconde Guerre mondiale, le phénomène associatif se développe de façon exponentielle. La notion de bonne œuvre s'estompe derrière l'action des associations créées à l'initiative de parents en réponse à l'attitude passive de l'État et l'incompréhension du reste de la population. L'État se mobilise alors davantage au plan financier et fait progressivement adopter des lois organisant la 
gestion morale et matérielle de la partie handicapée de son corpus social.

\section{$\underline{\text { Un virage inédit }}$}

Consultées, les associations deviennent une force de proposition qui intervient de plus en plus activement dans ce mouvement, en particulier dans les commissions influentes. En France, le premier tournant décisif dans l'histoire conceptuelle du handicap est amorcé le 30 juin 1975 avec la Loi $n^{\circ} 75-534$ d'orientation en faveur des personnes handicapées. Cette loi les valorise, essentiellement en tant que catégorie sociale. Elle est accompagnée le même jour par la Loi ${ }^{\circ} 75-535$ relative aux institutions sociales et médico-sociales. Trop muette sur les droits des personnes, cette dernière est réformée en 2002 par la Loi ${ }^{\circ}$ 2002-2 rénovant l'action sociale et médico-sociale. La parole est enfin accordée aux personnes concernées en tant que groupe partenaire au sein du Conseil de la vie sociale.

Un autre saut qualitatif est franchi le 11 février 2005. Il consacre la voix de l'individu et non plus celle de la catégorie sociale ou du groupe auxquels on l'a assimilé jusque là. Il ne s'agit plus seulement de demander à la personne son avis pour la signature d'une charte collective, mais de lui donner individuellement et prioritairement la parole pour l'élaboration de son projet de vie. Celui-ci doit émaner d'un échange permanent avec ceux qui l'entourent et repose sur la considération de ses capacités préservées. Il ne s'agit plus d'être entendu et écouté, mais d'être acteur. Le rôle de l'institution subit dès lors une mutation. Il lui faut vouloir et savoir comment faire exprimer les besoins et s'effacer devant l'autonomie individuelle tout en respectant les principes de précaution qui s'imposent. La qualité de vie n'est pas à restreindre sur la base de la compensation à l'accessibilité au seul environnement physique. Pour assurer le droit à la citoyenneté l'institution est censée intégrer l'accès à la sexualité dans une prise en charge globale et contractuelle quel que soit le degré de l'atteinte cognitive et l'inscrire dans les projets d'établissements dont elle dessine les orientations générales.

Or l'institution est souvent démunie face à des situations qui peuvent concerner aussi bien le respect de la pudeur au regard de la nudité dans les soins que l'explication des interdits dans le règlement intérieur ou le recueil du consentement éclairé. De plus, ses responsabilités se trouvent imbriquées dans le jeu des représentations qui peinent à changer (Gomez, 2005). En réalité, la question de l'accès à une vie affective et sexuelle en institution commence à poindre bien avant la période qui vient d'être évoquée : à partir des années 1960-1970, elle a bénéficié de l'évolution générale des mentalités liée à la révolution sexuelle qui a remis en cause le lien entre mariage et procréation pour valoriser une société $\mathrm{du}$ désir. Pour autant, les représentations de sexualité déviante ou d'asexualité vis-à-vis du handicap mental vont perdurer (Korff-Sausse, 2005). La perception du handicap sous l'angle d'un manque irrémédiable sévit encore aujourd'hui, accentuant l'asymétrie entre les positions du «bien portant», «fort et intelligent » et du malade, faible et déficient.

\section{Le retentissement sur les pratiques}

L'introduction de la mixité, l'ouverture sur l'extérieur, les abus sexuels, le développement des Maladies Sexuellement Transmissibles et le cadre législatif contraignent les institutions à traiter le problème par d'autres méthodes que la stérilisation forcée des femmes et la culpabilisation de la masturbation chez les hommes (Bajos, Bozon, Ferrand, Giami et Spira, 1998). Cependant, entre le droit de la personne à disposer d'elle-même (respect du corps, droit à l'intimité, à la vie privée, libre consentement) et le besoin de compenser sa fragilité par une protection assurée par la société, l'antagonisme s'affirme. Le milieu institutionnel tend à préserver ses responsabilités sous couvert du principe de précaution en occultant cette sexualité gênante pour un personnel exposé. Son acceptation bute sur des jugements de valeurs qui reflètent les malaises suscités dans le milieu professionnel. Aujourd'hui, la sexualité se vit sur les lieux d'hébergement souvent comme elle peut ou, à défaut, sur d'autres lieux de rencontre, comme par exemple les ateliers de travail ou les transports en commun. Les conditions de son vécu à l'abri des regards sont ainsi loin d'être toujours favorables (Vaginay, 2011).

Les interrogations concernent l'homosexualité, les comportements compulsifs à connotation sexuelle, la confidentialité, le secret professionnel et la dénonciation des infractions sexuelles, la prise en charge des victimes, l'harmonisation des approches dans et entre les établissements d'une même association gestionnaire, la place des 
parents et des proches. Elles sont vécues alors que la parole des principaux concernés n'est pas ou plus possible et risque d'être confisquée par les proches qui prennent en toute bonne foi le parti et l'habitude d'exprimer pour l'autre ce qui est bien pour lui. Le problème est accentué par le poids des parents - lorsqu'ils sont encore là non seulement lors du retour en famille, mais aussi de par leur implication officielle dans le fonctionnement institutionnel (participation au Projet de vie et au Conseil de la vie sociale).

À l'occasion des nouvelles vagues d'accréditation, cette part intime de l'individu commence à être inscrite par la force de la loi dans les projets d'établissements. Cependant, sauf exception conçue comme des réponses pragmatiques sans conceptualisation préalable (ex. création d'unité pour couple avec chambre double, réservation de chambre d'hôtel), elle n'y est formulée qu'en termes d'annonce. Faute de réflexion préalable et de moyens adaptés, elle est reléguée au second plan des priorités. Les crédits sont affectés à la mise en conformité des locaux pour la sécurité et l'accessibilité physique, à l'adaptation ergonomique des équipements, sans forcément offrir les conditions architecturales d'intimité que suppose la relation amoureuse sexuelle ou non. L'organisation des services privilégie les actes dévolus à l'hygiène et aux soins jugés plus urgents, aux dépens d'attentes qui exigent des qualités d'écoute moins figées, une capacité de remise en cause, un travail en équipe autrement coordonné et planifié. Ces besoins requièrent des compétences professionnelles et supposent des prérogatives qui renvoient à une redéfinition des métiers mobilisés.

\section{CONSTITUTION DU DOCUMENTAIRE DE BASE}

Pour mieux comprendre ce que la sexualité peut avoir de transversal dans la dépendance et de différent selon les pathologies, pour fédérer les réflexions et les actions isolées, un programme de recherche (SEXHI : SEXualité et Handicap en Institution) a été lancé. Soutenu par la Maison des Sciences de l'Homme Lorraine - USR-CNRS 3261, il a eu, entre autres objectifs, celui de construire un observatoire de la sexualité en institution auprès duquel les acteurs concernés peuvent trouver un étayage à leur réflexion, initiatives, attentes. Pour ce faire, il s'appuie sur la recension de documents. Il est enrichi de témoignages obtenus directement lors d'échanges individuels et collectifs avec 80 directeurs d'établissements, des personnels éducatifs et d'accompagnement de ces établissements, des parents rencontrés à l'occasion de séminaires de formation, de conférences-invité. Ces apports sont complétés par les règlements intérieurs et les chartes communiqués par une vingtaine de ces établissements, des exemplaires de contrats de séjours, de projets de vie anonymés et d'une analyse prospective par entretien du personnel d'un établissement (rapport de recherche non publié).

\section{Environnement logistique}

L'observatoire au cœur du travail ici présenté repose sur la constitution d'une base de données de type PHP/MySQL pour être interrogé par le biais d'un moteur de recherche bibliographique, et sur des moyens de sa mise à disposition et diffusion les plus larges auprès de tous les publics concernés ou intéressés. Techniquement parlant, cette base est consultable via un site Internet hébergé par la MSH Lorraine, élaboré par le service de communication de cette dernière. Le site Web est édité par un CMS (Content Management System) de Typo3 et propose des formulaires de contact et de dépôt avancé de documents par les utilisateurs.

La consultation du site a été ouverte au public fin 2009 à l'adresse : http://sexhi.msh-lorraine.fr. L'animation du site et le contrôle des contenus sont assurés par les membres du projet. Ce site est le portail dynamique d'un espace évolutif de ressources et de partage d'informations qui se solde actuellement par environ 1300 visites et 2100 pages consultées, avec un pic enregistré à l'occasion d'un colloque organisé par les partenaires du projet («Sexualités et Handicap en Institution », juin 2010). Cet outil doit être développé dans le cadre d'un second projet de recherche financé par la MSH Lorraine (Projet 2s2i : "Santé et Sexualité, Imaginaires et Institutions $»)$.

\section{Critères de structuration}

Les références portent sur la population des adolescents de plus de 15 ans (majorité sexuelle), des adultes handicapés de tous types d'établissements et selon toutes les formes de prises en charge, ainsi que celle des personnes 
âgées en Établissement d'Hébergement pour Personnes Âgées Dépendantes et Assimilées. Elles ont été recensées sur une période de plus de 35 ans. Les sources traitant de la sexualité en général ou d'un type de handicap sans aborder la dimension sexuelle ou affective ont été exclues de cette banque de données.

Les références issues de supports scientifiques proviennent de sept bases de données (PsycINFO, Science Direct, Medline \& Pubmed, Sudoc, Pascal, Francis, CAIRN), à partir des mots-clés suivants : Handicap, Handicap mental, Handicap moteur, Handicap physique, Handicap sensoriel, Handicap psychique, Personnes âgées, Vieillesse, Maladie mentale, Déficience, Sexualité, Intimité, Affectivité, Couple, Relations amoureuses, Institution, Établissement spécialisé. Elles ont été enrichies par les productions internes d'institutions, les documents juridiques disponibles, la collecte de jurisprudences, les actions et produits réalisés, l'identification des liens utiles et les ouvrages et articles de vulgarisation trouvés sur Internet ou transmis par différents organismes. Scientifiques ou non, ces références ont été retenues, quels que soient leur objectif et le public visé. Pour que chaque internaute puisse s'orienter dans leur exploration, elles ont été triées selon le type de documents et de populations concernées.

\section{Caractéristiques générales}

À l'heure de la finalisation de cet article, ce corpus général comprend 634 références francophones (Tableau 1). La grande majorité correspond à des articles (252) et à des ouvrages (156). Leur variété est renforcée par des manifestations d'envergure différente ouvertes au grand public souvent organisées par des associations : colloques, congrès, conférences, journées d'étude (87). Ce fonds inclut des sites Internet (40), des expériences, programmes et formations (34) et une proportion pratiquement équivalente de documents internes sous forme de rapports, chartes et recommandations (30). Il intègre les médias par les films et chroniques radio (26). Il comporte des textes juridiques (9 articles de lois et circulaires) (on note par ailleurs, des apports personnels très marginaux sous forme de trois poèmes).

\section{Tableau 1}

Ventilation de la typologie des ressources dans le corpus général

\begin{tabular}{lcc}
\hline \multicolumn{1}{c}{ Type de ressource } & Effectif & Pourcentage \\
\hline Articles & 252 & $39,75 \%$ \\
Ouvrages & 156 & $24,61 \%$ \\
Colloques & 87 & $13,72 \%$ \\
Internet & 40 & $6,31 \%$ \\
Expériences & 34 & $5,36 \%$ \\
Rapports & 30 & $4,73 \%$ \\
Films/Radios & 26 & $4,10 \%$ \\
Juridiques & 9 & $1,42 \%$ \\
\hline
\end{tabular}

La répartition de ces sources selon le type de population montre que les grandes catégories de dépendance sont représentées (Tableau 2). De nombreuses références s'intéressent simultanément à plusieurs populations citées expliquant un total cumulé de 688 références. 326 d'entre elles s'intéressent à la sexualité des personnes en situation de handicap mental, 209 concernent celle des personnes en situation de handicap physique, 88 évoquent la sexualité des personnes âgées dépendantes, 65 celle des personnes en situation de handicap psychique. Ainsi, la question rencontre de loin le maximum d'écho dans le domaine du handicap mental. Reste à savoir ce qui caractérise cette saillance et ce qu'elle peut signifier. 


\section{DOCUMENTATION SPÉCIFIQUE AU HANDICAP MENTAL}

Cette prééminence quantitative s'accompagne d'une singularité qualitative qui s'exprime au niveau de l'importance relative des supports et des modalités de communications (militante, professionnelle, recherche et journalistique) et de leur évolution temporelle. Cette double particularité est explicitée ci-dessous en termes de volumes catégoriels de production et de commentaire sur les contenus qui donnent sens à l'évolution qualitative.

\section{Tableau 2}

Répartition des ressources par catégorie de population

\begin{tabular}{lcc}
\hline \multicolumn{1}{c}{ Catégorie de population } & Effectif & Pourcentage \\
\hline Handicap mental & 326 & $47,38 \%$ \\
Handicap physique & 209 & $30,38 \%$ \\
Vieillesse & 88 & $12,79 \%$ \\
Handicap psychique & 65 & $9,45 \%$ \\
\hline
\end{tabular}

\section{Différentiel au niveau structurel}

Lorsque l'examen se focalise (Tableau 3) sur la structure de la documentation ciblant la population des personnes en situation de handicap mental (326 références), il permet de discerner une double tendance. Si certains supports sont moins bien représentés que dans le corpus général, d'autres se signalent de façon plus avantageuse.

$\underline{\text { Tableau } 3}$

Ventilation de la typologie dans la documentation ciblée

\begin{tabular}{lcc}
\hline \multicolumn{1}{c}{ Type de ressource } & Effectif & Pourcentage \\
\hline Articles & 134 & $41,10 \%$ \\
Ouvrages & 68 & $20,86 \%$ \\
Colloques & 56 & $17,18 \%$ \\
Internet & 6 & $1,84 \%$ \\
Expériences & 29 & $8,90 \%$ \\
Rapports & 21 & $6,44 \%$ \\
Films/Radios & 6 & $1,84 \%$ \\
Juridiques & 6 & $1,84 \%$ \\
\hline
\end{tabular}

Ainsi, articles et ouvrages sont majoritaires comme dans le corpus général $(61,96 \%)$. Cependant, les premiers sont en proportion pratiquement équivalente $(41,10 \%)$, alors que les seconds y figurent en moins grand nombre $(20,86 \%)$. La différence est également marquée par une très faible audience sur Internet $(1,84 \%)$ et un accès très réduit aux médias $(1,84 \%)$. En revanche, la sexualité est davantage abordée dans des colloques, congrès, conférences et journées d'étude $(17,18 \%)$, également dans des récits d'expériences, programmes de formations $(8,90 \%)$, rapports, chartes et recommandations $(6,44 \%)$.

Par rapport aux autres formes de dépendance, cette configuration montre qu'ici la documentation est orientée. Comme le laissent 
entendre les données brutes reportées dans le Tableau 4, la sexualité semble être l'objet d'un traitement différent, en particulier comparativement aux situations physiquement handicapantes. Dans la mesure où elles sont accessibles, il est légitime de penser que toutes les références procèdent d'une intention de communiquer. Les différences observées se situent au niveau de chacun des éléments qui fondent la communication (entités émettrice et réceptrice, canal, message et motivation). Les interlocuteurs potentiels sont des partenaires avec lesquels échanger, plus que des instances à sensibiliser ou à convaincre. Les canaux préférentiels (articles et expériences) reflètent l'intervention de professionnels soucieux de partager leurs incertitudes tant sur le plan des représentations que des pratiques. Le message est informatif et interrogatif plus qu'explicatif et démonstratif. La motivation est de trouver des réponses et pas de revendiquer des droits comme dans le cas du handicap physique où les personnes concernées sont intellectuellement aptes à défendre leurs droits, autonomie qui

Tableau 4

Distribution brute des types de document par catégorie de population

\begin{tabular}{lcccc}
\hline \multicolumn{1}{c}{$\begin{array}{c}\text { Nature des } \\
\text { supports }\end{array}$} & Handicap mental & Handicap physique & Vieillesse & $\begin{array}{c}\text { Handicap } \\
\text { psychique }\end{array}$ \\
\hline Articles & 134 & 58 & 48 & 23 \\
Ouvrages & 68 & 59 & 11 & 21 \\
Colloques & 56 & 39 & 11 & 9 \\
Expériences & 29 & 7 & 1 & 4 \\
Rapports & 21 & 6 & 4 & 1 \\
Films radio & 6 & 17 & 3 & 0 \\
Sites & 6 & 18 & 6 & 2 \\
Juridiques & 6 & 5 & 4 & 5 \\
Totaux & 326 & 209 & 88 & 65 \\
\hline
\end{tabular}

explique aussi leur accès facilité aux médias et aux techniques informatiques de communication.

\section{$\underline{\text { Différentiel au niveau temporel }}$}

D'un point de vue diachronique, un aperçu général (Figure 1) fait ressortir une période charnière autour des années 1990 avant laquelle la production est anecdotique, alors qu'après elle connaît une «flambée » marquée par des soubresauts réguliers. La documentation présente alors une alternance en dents de scie de pics et de ruptures comme autant de phases aiguës d'intérêt et d'autres promptes au silence, et qui dénote ainsi une évolution ni progressive ni régressive. Cette succession d'états signifie que la problématique ne s'inscrit pas dans une réflexion continue. Elle attire une attention qui semble chaque fois s'épuiser, comme si la mobilisation ne répondait que momentanément, de façon réactive, à des attentes ponctuelles. Les phases actives la valorisent comme un phénomène méritant qu'on s'y attarde, sans pour autant lui accorder une importance fondamentale. Le caractère itératif de ces épisodes contrastés trahit le manque de réponse satisfaisante. Chacun d'eux se résume à transmettre des messages sans opérer de changement chez leurs auteurs et leurs destinataires, contrairement à ce qu'implique toute communication aboutie. On cherche moins à transformer l'autre qu'à comprendre les données du problème, ce qui conduit à remettre sans cesse l'ouvrage sur le métier.

L'évolution différenciée des principaux types de production, tels que la littérature, les rapports et les colloques est également éloquente. Les deux dernières rubriques demeurent inexistantes ou rares pendant une période beaucoup plus longue que la première. Les trois semblent s'articuler ensuite pour entrer en phase avec le tempo des textes législatifs et réglementaires, de façon cependant décalée. Ces différences de périodicité signent la mutation des questionnements. En lien 
avec l'évolution de la législation dans le domaine de l'accompagnement des personnes en situation de handicap mental, ouvrages et articles précèdent, accompagnent et poursuivent l'élaboration, la parution, la mise en application de ces textes qui formalisent progressivement l'état des réflexions. Ils témoignent d'un glissement des préoccupations qui passe de l'explication des résistances à la recherche des moyens pour les réduire. Les tabous et les peurs

\section{$\underline{\text { Figure } 1}$}

Évolution temporelle des principaux types de production

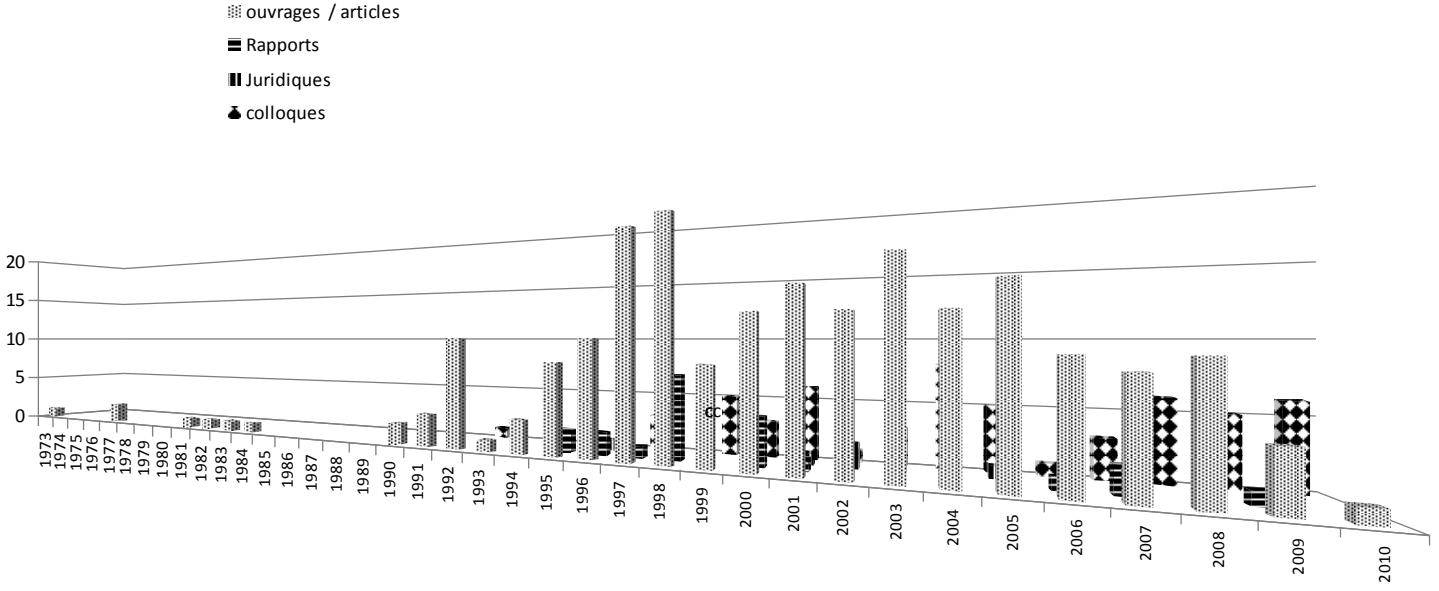

persistent, y compris les plus radicales du fait d'une attention trop crispée sur la génitalité et ses conséquences en termes de procréation. Néanmoins, les préoccupations s'ouvrent à une compréhension plus large de la sexualité.

\section{Accélération récente}

En ce qui concerne les ouvrages et articles, trois étapes en jalonnent l'avancée. Avant les années 1970, le faible intérêt pour la sexualité des personnes handicapées mentales considérées comme «hors normes" traduit un déni de la réalité. En fait, la question se pose peu à cette époque. La période qui voit poindre une première et modeste attention se situe entre 1973 et 1991.
Alors que la loi de 1975 se prépare et se trouve promulguée, la production qui entoure son avènement n'est pas flagrante. Si elle amorce le

virage conceptuel du handicap, elle n'entraîne pas non plus d'augmentation dans les années qui suivent. Le temps n'est pas encore venu de provoquer un débat autour de la problématique. Encline à changer le regard sur le handicap, cette loi s'attaque à un rempart de représentations qui dépassent largement la sexualité. Dans des publications qui demeurent marginales (12), la sexualité commence à être abordée sous les angles médical et sociologique, et sous celui des risques. Y sont rapportées les questions de contraception, de stérilisation, d'abus sexuels, d'actes pédophiles et une réflexion sur les représentations. 
C'est à partir de 1992 qu'ouvrages et articles se multiplient (79 références). Un pic est atteint suite à la circulaire de 1996 sur la prévention de l'infection à VIH dans les services accueillant des personnes handicapées mentales. Avant cette date, les discours restent limités à la reconnaissance du besoin affectif. Ensuite, ils revendiquent ouvertement ce besoin en termes de qualité de vie, mais en y incluant sa déclinaison sexuelle, en affirmant le droit à l'éducation sexuelle. Le thème des représentations sociales du handicap demeure présent, mais de nouvelles préoccupations émergent, telles que la stérilisation abusive, la prévention des maladies sexuellement transmissibles, la vie affective et la parentalité. De plus, l'entourage de la personne en situation de handicap mental est pris en compte. Des guides sont conçus à son intention. Cette augmentation de la production est concomitante des actions associatives de plus en plus nombreuses. Si la prise de conscience des risques à ce niveau est acquise sous le coup de la crainte de la contamination, elle s'enracine peu dans les faits.

\section{INDICATEURS D'ÉVOLUTION}

L'analyse croisée des différents types de supports et de leur évolution temporelle laisse transparaître quelques éléments qui permettent d'anticiper l'avenir de la problématique. Avec une fréquence plus élevée, mais pas toujours forcément soutenue, la production accroît avec le temps sa visibilité et favorise la lisibilité de ses grands axes.

\section{Mutation des préoccupations}

De 2000 à 2010, une vague de production (111 références) porte sur l'éducation et l'accompagnement à la sexualité. Ouvrages et articles traitent de l'accompagnement érotique et sexuel, parallèlement à la reconnaissance du statut d'assistant sexuel dans les pays francophones. Cette recrudescence peut également s'expliquer par l'évolution législative. En 2002, la Loi de rénovation et modernisation de l'action sociale marque un pas. Elle est censée valoriser les personnes en situation de handicap, notamment en favorisant dans un souci de normalisation sociale leur insertion scolaire, sociale et professionnelle à la mesure de leurs moyens. L'amplification se ralentit ces cinq dernières années, mais avec des préoccupations qui concernent davantage l'intimité, l'affectivité, les dimensions du désir et du plaisir sexuel, la parentalité cadrant ainsi davantage avec la loi de 2005. Ce changement traduit la modification progressive des représentations sociales et des prises en charge en institution. Il se formalise dans les termes utilisés qui ne sont plus aussi négatifs qu'auparavant. Les titres qui contenaient des mots comme «maladie», « abus», «violence ", "stérilisation" font place à ce vocabulaire plus positif.

En majorité français (155), les ouvrages et articles (47 soit plus de $23 \%$ du corpus) sont également publiés en Suisse, en Belgique, au Canada, pays dans lesquels l'assistance sexuelle est instaurée ou des programmes d'éducation sexuelle sont élaborés. Les ouvrages sont le fait d'auteurs prolixes et actifs dans le domaine ou d'associations militantes. Nombre de ces ouvrages sont composés de revendications et de témoignages de personnes directement ou indirectement concernées par le handicap. Les titres reflètent leur contenu militant, pour illustration: "Oui, ce sont des hommes et des femmes $[. .]$.$» (Beyer Zilliox, Clere et Duchamp,$ 2002), «Nous respecter, c'est vous respecter [...]» (Destexhe, 2002). Le handicap mental occupe quelques chapitres dans cet environnement qui le dépasse. Les articles sont publiés dans plus de 50 revues différentes. Leurs intitulés sont caractéristiques des préoccupations passées et actuelles des associations, avec souvent un souhait de dénoncer des pratiques discriminantes, pour exemple : «Moi aussi je veux un bébé » (Agthe Diserens, 1998), « Faut-il stériliser tous les affreux? » (Diederich, 1999). La plupart des supports sont des bulletins d'associations, des revues religieuses ou politiques. La recherche dans les bases de données n'a permis de recenser qu'une trentaine de références scientifiques qui relèvent essentiellement de revues spécialisées pour un public averti de travailleurs sociaux et/ou orientées vers le handicap.

\section{$\underline{\text { Diversification des auteurs }}$}

Ouvrages et articles sont l'œuvre de plus de 150 auteurs de formations diverses (médecins, sociologues, psychologues, philosophes et sexologues) dont une dizaine se démarquent par leur productivité et constituent des pôles dynamiques de production. La diversité de leurs 
propos est le reflet de la pluridisciplinarité du champ. La concentration exprime le fait que parmi les principaux auteurs, plusieurs sont euxmêmes en situation de handicap, notamment moteur et/ou sensoriel et fortement engagés dans le mouvement associatif ou militant. Les autres sont nombreux à présider des associations ou à y participer en tant que membres en vue de défendre les droits des personnes en situation de handicap et de promouvoir leur vie affective et sexuelle.

Cette production est également signée par des associations, telles que l'Association genevoise de Parents et d'Amis de Personnes Mentalement Handicapées, INSIEME (Fédération suisse regroupant 50 associations de parents), l'Union Nationale des Associations de Parents de personnes handicapées mentales et de leurs amis (UNAPEI), rappelant par là même leur détermination historique concrétisée par la création de nombreux établissements. Elle est également relayée par des organismes tels que les Centres Régionaux d'Études et d'Actions sur les Handicaps et les Inadaptations dont la vocation est de faire avancer la réflexion en articulant démarche de recherche et questions d'actualité.

\section{Modulation des modes de communication}

À partir des années 2000, un nouveau mode de communication se développe sous forme de colloques, congrès, conférences, et journées d'étude (56). Il progresse de façon plus importante que dans le reste de la dépendance (soit 17,18\% de l'ensemble de la production contre $13,72 \%$ ). Alors qu'on ne repère aucune manifestation de ce type avant 1992, on en relève 9 de 1992 à 1999, puis 47 de 2000 à 2010. Pour la plupart organisées à l'initiative d'associations, ces manifestations témoignent de leur souci d'ouverture et de convergence, et au-delà de leur histoire de leurs politiques respectives et de leur répartition géographique diffuse. Elles font preuve de leur volonté à faire avancer concrètement la question en informant l'opinion et les pouvoirs publics, en ouvrant le médical au social, en sensibilisant les personnels et les parents. Non restreintes aux publics d'initiés, elles visent ainsi à élargir et mobiliser l'audience, en jouant sur la proximité pour inciter les intéressés à participer et en leur donnant la parole au sein d'ateliers.
Leurs organisateurs comptent sur l'opportunité des contacts pour dynamiser les échanges. Plus rapides que la rédaction d'écrits à coïncider avec l'actualité, plus adaptées au temps qui presse, ces manifestations n'échappent cependant pas au risque d'éparpillement, de redondance et de réactivité à courte visée. Malgré ce risque, elles présentent un intérêt non négligeable. Contrairement aux autres formes de production ou plus souvent qu'elles (bien plus que les ouvrages), elles intègrent toutes les formes de handicaps autour d'une même cause, celle de la sexualité - sans pour autant fédérer ceux qui les représentent - . Le handicap physique y côtoie le handicap mental ainsi libéré du double ostracisme auquel il est soumis : celui issu d'une population générale qui a du mal à le comprendre et celui provenant du handicap physique qui se méfie à juste raison - de l'amalgame dont il est souvent victime.

Le nombre d'expériences, programmes et formations est faible (29) comparé à celui des publications qui dénoncent les pratiques et proposent des "solutions » pour favoriser la vie affective, intime et sexuelle et améliorer la qualité de vie. Ces interventions se sont multipliées dans les années 2000, souvent à l'initiative d'associations ou d'établissements suisses et québécois. On y trouve des formations présentielles, dactylographiées, audiovisuelles et des groupes de parole. Certaines sont conçues pour être soumises aux personnes en situation de handicap mental (Mercier et Merlin, 2000; Dupras, 1999), d'autres sont destinées à leur entourage (familles et/ou professionnels). Le contenu est sensiblement le même : éducation sexuelle et affective dans les établissements français, accompagnement à la vie érotique et sexuelle dans les établissements étrangers. Les auteurs sont ceux qui se démarquent par leur grand nombre de publications.

Le nombre de rapports, chartes et recommandations est relativement important (21 références). Le thème est abordé dans de rares films et vidéos, supports qui au fil du temps ne se développent pas. En revanche, de nombreux sites Internet et des forums abordent le sujet. À titre indicatif, six sites Internet ont été recensés, notamment ceux des grandes associations. Ces dernières années, des blogues sont apparus où les individus publient leur témoignage. Ces références montrent que les actions se multiplient et qu'elles visent des objectifs et des aspects plus 
spécifiques, comme des sites Internet de rencontre, des sites féministes ou l'homosexualité. L'an 2000 marque l'émergence d'un nombre important de publications du mouvement militant et de textes juridiques qui s'attachent aux discriminations liées au handicap, à l'éducation sexuelle, aux abus sexuels, à la maltraitance dans les structures spécialisées.

\section{DISCUSSION-CONCLUSION}

Enjeu clinique et sociétal, la problématique de la sexualité des personnes en situation de handicap mental repose encore aujourd'hui sur une assise théorique essentiellement axée sur un modèle biologique ou génital (Giami, 2005). Or, sa portée dépasse ces bornes conceptuelles. Le parti a été pris d'appréhender cette complexité à travers la nébuleuse de productions par laquelle la question est accessible en évitant d'y faire un tri, dans la mesure où toutes sont susceptibles de revêtir une utilité pour ceux qui les parcourent. Par leur vocation informative et communicative, cette démarche et l'outil sur lequel elle s'appuie peuvent ainsi prétendre participer à la prise de conscience individuelle et collective de l'actualité du problème, fédérer, impulser les intérêts et les initiatives. L'aspect diachronique présente l'avantage de dégager le tempo de l'évolution de la problématique, de faire valoir ce qui a été fait et de dessiner les lignes du chemin qui reste à parcourir.

Cet avantage s'arrête néanmoins aux bornes que pose toute approche globale, là où débute la singularité des situations qui limite la portée du partage d'expérience. Or, cette singularité est à la fois l'une des caractéristiques de la problématique et l'une des difficultés majeures à surmonter dans la mesure où elle surgit à la croisée de représentations, responsabilités, attentes divergentes, de la direction, du personnel, des parents et des intéressés. L'évolution des pratiques et leur étude se heurtent à l'acceptation de toutes ces parties. Ainsi, la sexualité peut être reconnue par une Assemblée Générale et être inscrite dans le règlement intérieur, sans être pour autant mise en application. L'inverse existe aussi. Ce constat montre que l'on gagne à accompagner l'investigation par un investissement sur le terrain qui permet de circonscrire la diversité des points de vue. Par ailleurs, une telle approche nécessite un suivi de la documentation et de son analyse comme baromètre de son évolution. Elle requiert également de s'ouvrir à l'interactivité pour faire de l'outil une plateforme non pas prestataire de solutions, mais d'échanges pour mettre en relation concepteurs et utilisateurs (ce suivi et cette ouverture sont cours de réalisation grâce à la prolongation du projet SEXHI).

La documentation jusqu'ici répertoriée est en grande partie issue des milieux médico-social et associatif au sein desquels elle trouve un auditoire attentif. Son contenu montre comment l'articulation des lois et des mentalités s'inscrit dans les pratiques, fait valoir les difficultés à penser et à faire. Il témoigne du poids de conditions qui semblent souvent se liguer pour faire obstacle à la sexualité en tant qu'accomplissement. Les signes d'évolution sont néanmoins perceptibles. Les intérêts se mobilisent (tangibles par exemple par la demande d'aide, d'interventions, de formations au sein des établissements), même si c'est encore par à-coups et de façon isolée. Il est cependant difficile d'en évaluer les répercussions, car l'ambiguïté persiste entre les problèmes que la sexualité est susceptible de provoquer et ce qu'elle recouvre en termes de besoins et de ressentis chez les intéressés. La confusion semble perdurer entre incertitude et inquiétude.

Si une législation est capable aujourd'hui de mettre l'individu au cœur des dispositifs d'accompagnement, la question reste ouverte de savoir comment faire pour qu'il puisse connaître et comprendre ses droits. Les personnes concernées ne sont pas aptes à les défendre en raison de leurs facultés intellectuelles amoindries sur le plan de l'idéation, la compréhension et l'expression. La réponse doit s'appuyer sur toutes les facettes verbales et non verbales de la communication, la promotion $\mathrm{du}$ travail en équipe, la modification des représentations, la formation dans le respect de la déontologie, de la singularité au sein d'une population hétérogène aux facultés d'apprentissage réduites et labiles.

Convaincus ou contraints, ceux qui se penchent sur la question admettent que la sexualité ne peut être appréhendée qu'en intégrant la responsabilité d'autrui. La question est légitimement posée de savoir si, imprégnée d'intimité, la sexualité, est possible en collectivité au regard d'une société qui cherche de plus en plus à se prémunir des risques dont elle ne supporte pas le caractère imprévisible. La prise en compte de la sexualité 
en institution semble relever d'une question indécidable que le handicap mental exacerbe pour en faire un modèle du respect de la dignité dans ce type d'environnement (Marc, 2005).

Aujourd'hui, en France, le contexte est devenu porteur à la fois d'espoir et de danger (Zribi et Sarfaty, 2008). Un flambeau de revendication est actuellement porté par les militants représentatifs du handicap physique (Nuss, 2008). Le Comité Consultatif National d'Éthique a été saisi de la question pour l'aborder dans un premier temps par le biais de cette forme de handicap. Dans son rapport de 2010, le Conseil National Consultatif des Personnes Handicapées insère ses réflexions en chapitre 8 sur la vie affective, l'intimité, l'exercice de la sexualité et la parentalité (pp 8788). Il «livre au débat l'état actuel de ses réflexions quant à la question de l'accompagnement sexuel des personnes handicapées ». Cependant, la structuration en 1996 de l'hospitalisation publique et privée en Agences Régionales de l'Hospitalisation, avec récemment celle du secteur de la santé en Agences Régionales de Santé et leurs compétences élargies aux structures médicosociales, le champ de la santé semble s'affirmer à nouveau face à la démédicalisation progressive du handicap depuis une trentaine d'années. Dans un tel contexte, cette part intime de l'individu risque alors de paraître bien dérisoire, alors même que l'actualité voit se renforcer de plus en plus des demandes fortes en provenance du terrain et que les choses bougent aux frontières.

Aujourd'hui, il ne s'agit plus seulement de convaincre que la sexualité est présente dans le handicap mental comme force d'épanouissement et qu'à titre humaniste il faut la respecter. Il convient de mettre en œuvre les conditions pour qu'a minima elle ne soit pas brimée et qu'au maximum elle puisse s'exprimer. Éthiquement parlant, si les représentations évoluent relativement aux personnes handicapées mentales, on peut penser qu'elles modifieront a fortiori celles qui affectent les autres secteurs du handicap pour lesquels les problèmes se posent moins en termes de consentement que d'accessibilité. Faire avancer la réflexion dans ce champ du handicap contribue à faire sortir de l'ombre des préoccupations qui permettront de revisiter, au-delà de la sexualité et de l'intimité, les notions d'intégrité, d'autonomie, de bien être, et ce, pour des populations affectées d'autres pathologies, voire indemnes de toute atteinte pathologique.

\title{
MENTAL HANDICAP, SEXUALITY AND INSTITUTION: A MACRO-ANALYSIS OF FRANCOPHONE INFORMATION
}

\begin{abstract}
The issue of sexuality of people with intellectual disabilities declines in terms of individual behaviors, professional practices and organizational processes. Many disparate and heterogeneous published sources reflect both the urgency of the concern, based primarily on practical experience, and the difficulty to manage conceptual and regulatory changes toward effective answers. To reduce inherent problems resulting from the multiplicity of sources, an analysis of French-speaking literature related to mental disability is applied to a computerized set of documents collected on the occasion of interdisciplinary research projects. This analysis highlights the difficulties that persist in the implementation of solutions, despite some signs of progress.
\end{abstract}

\section{RÉFÉRENCES}

Agthe Diserens, C. (1998). Moi aussi je veux un bébé. INSIEME, 3.

Agthe Diserens, C., Mercier, M., Vatre, F. (2002). Éléments pour une éthique de l'intervention en éducation affective et sexuelle auprès des personnes vivant avec un handicap mental. Revue francophone de la Déficience Intellectuelle, 1 (13), 8192.

Bajos, N., Bozon, M., Ferrand, A., Giami, A., Spira, A. (1998). La sexualité au temps du 
sida. Presses Universitaires de France: Paris.

Bernardet, P. (2004). Les conditions du consentement de la personne souffrant de troubles mentaux. Handicap, revue de sciences humaines et sociales, 104, 35-55.

Beyer Zilliox, H., Clere, D., Duchamp, A. (2002). Oui, ce sont des hommes et des femmes: accompagnement de l'adulte handicapé mental très dépendant. L'Harmattan : Paris.

Brown, H. (1997). Sexualité, vie affective et déficience mentale. De Boeck : Bruxelles.

Delfosse, M. L., Delville, J., Duchene, J., Mattys, M., Mercier, M., Witd, O. (1997). Éthique et handicap mental. Presses Universitaires de Namur : Namur.

Delville, J., Mercier, M. (dir.) (1997). Sexualité, vie affective et déficience mentale. De Boeck : Bruxelles.

Destexhe, A. (2002). Nous respecter, c'est vous respecter: pour une approche civique de la personne handicapée. Luc Pire: Bruxelles.

Diederich, N. (1999). Faut-il stériliser tous les affreux? Ou : le droit à la parentalité en question. Lien social, 471.

Diederich, N., Printz, O. (2000). Quelle sexualité pour les personnes handicapées mentales? Lien social, 557.

Dupras, A. (1998). La désexualisation de la personne handicapée ou le syndrome de Forest Gump. Revue européenne $d u$ handicap mental, 18 (5), 47-52.

Dupras, A. (1999). La promotion de la qualité de vie sexuelle des personnes handicapées mentales vivant en institution : un cadre de référence pour un projet éducatif. Handicap, revue de sciences humaines et sociales, 83, 63-78.

Gascon, H. (2006). Vie affective et sexuelle des personnes présentant une déficience intellectuelle: défis et perspectives. In M. Mercier, H. Gascon, G. Bazier, Vie affective, relationnelle et sexuelle des personnes déficiences mentales (pp. 3346). Namur: Presses Universitaires de Namur.

Gardou, C. (dir.) (2004). Désinsulariser le handicap. Quelles ruptures pour quelles mutations culturelles? Erès : RamonvilleSaint-Agne.

Gasser, J., Heller, G., Jeanmonod, G. (2004). Règlementation de la stérilisation des personnes mentalement déficientes: perspectives historiques et réflexions actuelles. Revue européenne du handicap mental, 28, 15-28.

Germain, B., Langis, P. (1990). La sexualité, regards actuels. Études vivantes: Montréal.

Giami, A., Humbert, C., Laval, D. (2001). L'ange et la bête: représentations de la sexualité des handicapés mentaux par les parents et les éducateurs. CTNERHI : Paris.

Giami, A. (2002). Sexual health: the emergence, development and diversity of a concept. Annual Review of Sex Research, 13, 1-35.

Giami, A. (2005). Santé sexuelle: la médicalisation de la sexualité et du bienêtre. Comprendre. Revue de philosophie et de sciences sociales, 6, 97-115.

Giami, A., De Colomby, P. (2008). Relations sociosexuelles des personnes handicapées vivant en institution ou en ménage : une analyse secondaire de l'enquête Handicap, Incapacités, Dépendances. Alter, 2, 109132.

Gomez, J. F. (2005). Handicap, éthique et institution. Dunod : Paris.

Kerbage, H., Richa, S. (2011). À bord de la vie affective et sexuelle des déficients intellectuels. Neuropsychiatrie de l'enfance et de l'adolescence, 59, 478483.

Korff-Sausse, S. (2005). La notion de responsabilité dans le domaine du handicap. Autonomie, réciprocité et sexualité. Reliance, 18, 35-115. 
Lavigne, C. (1996). Entre nature et culture : la représentation de la sexualité des personnes handicapées mentales. Handicaps et inadaptations, 72, 58-70.

Marc, I. (2005). Liberté et responsabilité dans la vie affective, familiale et sexuelle en situation de handicap. Reliance, 18, 35-38.

Mercier, M. (dir.) (2004). L'identité handicapée. Presses Universitaires de Namur : Namur.

Mercier, M., Merlin, C. (2000). Des hommes et des femmes, programme d'éducation affective, relationnelle et sexuelle destiné aux personnes déficientes mentales. Presses Universitaires de Namur : Namur.

Nuss, M. (dir.) (2008). Handicaps et sexualités, le livre blanc. Dunod : Paris.
Rabischong, P. (2008). Le handicap. Presses Universitaires de France : Paris.

Vaginay, D., Balvet, D. (2002). Vivre la sexualité. Encyclopédie illustrée. Chronique sociale : Lyon.

Vaginay, D. (2006). Comprendre la sexualité de la personne handicapée mentale. Chronique sociale : Lyon.

Vaginay, D. (2011). Sexualité et handicap mental. Lois, majorité et consentement. In P. Pitaud et M. - F. Dubois-Sacrispeyre, Sexualité, handicaps et vieillissement (pp. 157-180). Toulouse : Éditions ERES.

Zribi, G., Sarfaty, J. (dir.) (2008). Handicapés mentaux et psychiques : vers de nouveaux droits. EHESP : Rennes. 